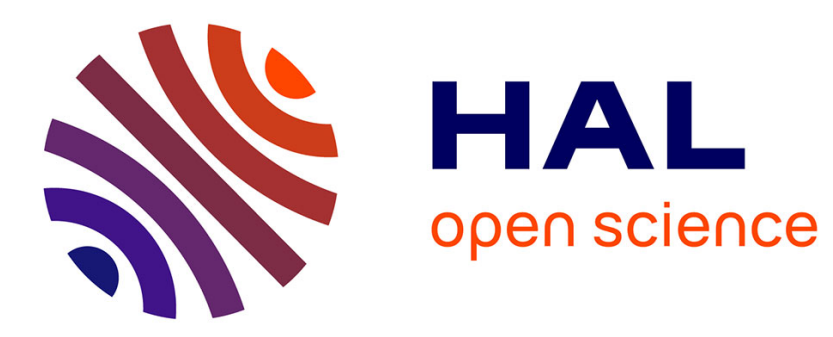

\title{
Simulations de l'interaction entre le catamaran Delft 372 et la houle à l'aide du couplage SWENSE-Level Set
} Gabriel Reliquet, Marie Robert, Lionel Gentaz, Pierre Ferrant

\section{To cite this version:}

Gabriel Reliquet, Marie Robert, Lionel Gentaz, Pierre Ferrant. Simulations de l'interaction entre le catamaran Delft 372 et la houle à l'aide du couplage SWENSE-Level Set. La Houille Blanche - Revue internationale de l'eau, 2019, 5-6, pp.59-66. 10.1051/lhb/2019030 . hal-02884903

\section{HAL Id: hal-02884903 \\ https://hal.science/hal-02884903}

Submitted on 8 Oct 2020

HAL is a multi-disciplinary open access archive for the deposit and dissemination of scientific research documents, whether they are published or not. The documents may come from teaching and research institutions in France or abroad, or from public or private research centers.
L'archive ouverte pluridisciplinaire HAL, est destinée au dépôt et à la diffusion de documents scientifiques de niveau recherche, publiés ou non, émanant des établissements d'enseignement et de recherche français ou étrangers, des laboratoires publics ou privés. 


\title{
Simulations de l'interaction entre le catamaran Delft 372 et la houle à l'aide du couplage SWENSE-Level Set
}

\author{
Gabriel Reliquet ${ }^{1, *}$, Marie Robert ${ }^{1,2}$, Lionel Gentaz ${ }^{1}$ et Pierre Ferrant ${ }^{1}$ \\ ${ }^{1}$ Laboratoire de recherche en Hydrodynamique, Énergétique et Environnement Atmosphérique (LHEEA), UMR CNRS 6598, \\ École Centrale de Nantes, France \\ ${ }^{2}$ HydrOcean, Nantes, France
}

\begin{abstract}
Résumé - Ce papier présente les derniers développements concernant le couplage de la méthode SWENSE (Spectral Waves Navier-Stokes Equations) et d'une méthode de résolution des équations RANS (Reynolds Averaged Navier-Stokes) avec capture d'interface de type Level Set. Ce couplage permet de combiner les avantages des deux méthodes, c'est-à-dire avoir une cinématique de houle de bonne qualité dans tout le domaine grâce à la méthode SWENSE et la prise en compte du déferlement via la fonction Level Set. Le catamaran Delft 372 est utilisé pour les validations avec des calculs sur mer calme et sur houle régulière à deux vitesses différentes.
\end{abstract}

Mots clés : CFD / SWENSE / Level Set / catamaran

Abstract - Simulations of the Delft $\mathbf{3 7 2}$ catamaran in waves using SWENSE-Level Set coupling. This paper presents recent developments around the combination of the SWENSE (Spectral Waves Navier-Stokes Equations) method and a method solving RANS (Reynolds Averaged Navier-Stokes) equations with a free surface-capturing scheme. Such coupling allows us to use the best of both methods: high quality regular or irregular waves in the entire domain using a spectral potential flow solver with a small CPU cost for SWENSE and the possibility to modelize the wave breaking using a single-phase Level Set free surface capturing scheme. In this work, the Delft 372 catamaran is used for all validations in calm water and in regular waves for two different speeds.

Keywords: CFD / SWENSE / Level Set / catamaran

\section{Introduction}

Aujourd'hui, plus que jamais, l'objectif est de simuler numériquement les configurations les plus réalistes possibles. Toutefois, la modélisation complète et réelle de l'écoulement autour d'un navire reste difficile car prendre en compte à la fois un état de mer réel, les appendices, les efforts aérodynamiques, la structure du navire est beaucoup trop complexe et gourmand en temps de calcul. Bien souvent, il est possible de résoudre un ou plusieurs de ces phénomènes tout en simplifiant les autres.

$\mathrm{Au}$ sein de l'équipe Hydrodynamique, Interfaces et Interaction (H2I) du LHEEA, différents codes sont développés afin de traiter de manière complémentaire les principaux problèmes rencontrés en hydrodynamique. Pour traiter les problèmes de tenue à la mer (interaction houle-carène), la méthode SWENSE, développée au sein du LHEEA, a montré

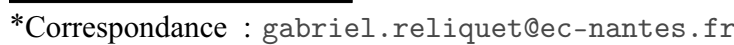

son intérêt, permettant de réaliser des simulations de navire sur houles irrégulières complexes avec une faible augmentation du temps de calcul par rapport à un calcul sans houle. Proposée par Pierre Ferrant (Ferrant et al., 2003), la méthode est basée sur une décomposition fonctionnelle du problème en une partie incidente obtenue à partir d'un modèle de propagation de houle en fluide parfait potentiel et d'une partie diffractée obtenue par la résolution des équations de Navier stokes modifiées (où le champ incident est vu comme un forçage). Cette approche a été validée en suivi de surface libre par 2 thèses (Luquet, 2007 ; Monroy, 2010). L'utilisation de cette décomposition dans des codes de calcul où la surface libre est mise à jour via une méthode de capture d'interface n'est pas immédiate mais cela a été fait au sein du LHEEA dans le code ICARE (Reliquet, 2013) et dans Openfoam par l'université de Zagreb (Vukčević et al., 2016). Avec une résolution diphasique eau-air, la question du champ incident imposé dans l'air se pose et c'est pourquoi des différences notables peuvent être observées quand on compare les 
différentes formulations de SWENSE existantes. En effet, les modèles utilisés pour les houles régulières et irrégulières sont la plupart du temps des modèles spectraux basés sur la théorie potentielle permettant une évaluation de l'écoulement n'importe où (même au-dessus de la surface libre). Toutefois, il faut garder en tête que le champ incident évolue exponentiellement avec l'altitude et devient rapidement non physique si la hauteur maillée est trop importante et c'est pourquoi il est important de modifier le champ incident dans l'air en fonction des spécificités du code de calcul.

Le modèle utilisé dans le code ICARE a été validé avec succès pour le cas de la frégate militaire DTMB 5415 sur des houles régulières et irrégulières (Reliquet et al., 2014) et ce papier présente les dernières validations réalisées avec le catamaran Delft 372. Cette carène a été retenue car elle a fait l'objet de nombreux essais aussi bien sur mer calme que sur houles régulières ainsi que de simulations numériques par d'autres auteurs (Castiglione et al., 2011; He et al., 2015; Visonneau et al., 2012).

\section{Développements}

Le code de calcul utilisé (Drouet, 2011 ; Reliquet, 2013) est basé sur le code ICARE développé au sein du LHEEA de l'École Centrale de Nantes depuis une vingtaine d'années. Les équations RANS sont discrétisées à l'aide de schémas aux différences finies d'ordre 2 en espace et en temps après une transformation partielle des équations permettant de passer du repère cartésien à un repère curviligne adapté au corps, tout en conservant les variables vectorielles exprimées dans le repère cartésien. Avec une résolution monophasique (eau uniquement), les inconnues résolues sont les composantes de la vitesse $\left(U_{j}\right)$, la pression dynamique $(P=p+\rho g z)$, la variable liée à la surface libre (surfacique en suivi de surface libre et volumique avec un modèle de capture d'interface) et les variables liées au modèle de turbulence (ici l'énergie cinétique turbulente $k$ et le taux de dissipation spécifique $\omega$ ). Un positionnement entrelacé partiel des inconnues est adopté, c'est-à-dire que les variables de pression et Level Set sont localisées aux centres des cellules tandis que les autres variables sont positionnées aux nœuds du maillage. Ce positionnement présente l'avantage de ne pas avoir à utiliser de conditions aux limites pour la pression sur le corps. La turbulence est modélisée par le modèle $k-\omega$ de Wilcox (Wilcox, 1988).

Quant à la surface libre, elle est modélisée à l'aide d'une fonction Level Set définie comme la distance signée à l'interface. Cette approche a été préférée à la méthode VOF (Volume of Fluid) car il s'agit d'une fonction continue qui ne nécessite donc pas l'utilisation de schémas spécifiques pour la convection.

$$
\phi=\left\{\begin{array}{ccc}
d \text { dans } & 1 / \text { air } \\
-d & \text { dans } & 1 / \text { air }
\end{array} .\right.
$$

Afin de conserver une résolution proche de celle qui était utilisée en suivi d'interface dans ICARE, une approche monophasique a été retenue. Ainsi, l'air est maillé mais non modélisé. Seules les vitesses seront calculées dans l'air par extrapolation afin de pouvoir mettre à jour la fonction Level Set. Afin de faciliter le traitement, celle-ci est décomposée en

\begin{tabular}{|c|c|c|c|c|}
\hline 0 & 0 & 0 & 0 & 0 \\
\hline 0 & 0 & 0 & 0 & 0 \\
\hline 0 & 0 & 0 & 0 & 0 \\
\hline 0 & 0 & 0 & 0 & 0 \\
\hline 0 & 0 & 0 & 0 & 0 \\
\hline 0 & 0 & 0 & 0 & 0 \\
\hline
\end{tabular}

Fig. 1. Description du traitement effectué en fonction de la position des nœuds et des centres des cellules par rapport à la surface libre.

3 champs : le champ initial correspondant à la Level Set à $t=0$ sans corps (altitude $z$ ), le champ incident correspondant à l'ajout de la houle $h_{i n}$, et le champ diffracté $\left(\phi^{\prime}\right)$ correspondant à la perturbation liée à la présence du corps et/ou de la houle.

$$
\phi=\phi \prime+z-h_{i n}
$$

Cette décomposition permet d'initialiser simplement le champ diffracté Level Set à zéro, quelle que soit la simulation. L'équation de convection résolue sera alors modifiée pour prendre en compte cette décomposition, soit:

$$
\frac{\partial \phi \prime}{\partial t}+\left(U_{1}-V_{g}^{1}\right) \frac{\partial \phi \prime}{\partial x_{1}}+\left(U_{2}-V_{g}^{2}\right) \frac{\partial \phi^{\prime}}{\partial x_{2}}=-U_{3}
$$

avec $V_{g}^{i}=\frac{\partial x_{i}}{\partial t}$ désignant la vitesse de glissement du maillage et $U_{3}$ la vitesse verticale.

Dès lors et comme cela a déjà été évoqué dans les papiers précédents (Reliquet et al., 2014), la difficulté pour la discrétisation du système pression-vitesses vient du positionnement entrelacé partiel que l'on a choisi de conserver. En effet, il est impossible de conserver un traitement physique à la fois de la pression et la vitesse au voisinage de l'interface. Ainsi, l'équation de pression est obtenue classiquement via une reconstruction de Rhie et Chow (1983) sous la surface libre et on imposera la condition dynamique normale aux centres des cellules ayant un voisin au-dessus de la surface libre. Pour les autres inconnues de pression, la pression est imposée à zéro (Fig. 1).

Pour les vitesses, le traitement est plus simple. Si un nœud est sous la surface libre et si les huit centres des cellules entourant le nœud sont dans l'eau, alors les équations de conservation de la quantité de mouvement sont résolues. Dans le cas contraire, la vitesse est propagée à l'aide de la normale obtenue à partir de la Level Set $\left(\left(\vec{n}=\frac{\vec{\nabla}(\phi)}{\|\vec{\nabla}(\phi)\|}\right)\right)$ comme suggéré par Adalsteinsson et Sethian (1999).

\subsection{Traitement de la condition dynamique normale}

Par rapport aux développements présentés précédemment, le principal développement lié à la discrétisation du système vitesses-pression concerne le traitement de la condition dynamique normale. Auparavant, la pression était calculée à partir des deux cellules qui étaient le plus alignées avec la normale à l'interface (Fig. 2). Pour des raisons de stabilité, la pression au centre d'une cellule est maintenant calculée en prenant en compte l'ensemble des couples entre lesquels passe 
la surface libre en pondérant leur influence par la distance à l'interface.

\subsection{Gestion des topologies multi-blocs}

Depuis quelques années (Drouet, 2011), le code de calcul prend en charge les topologies multi-blocs. Le maillage n'est donc plus constitué d'un seul bloc que l'on déforme afin d'épouser la géométrie mais de plusieurs blocs dont certains seront toujours déformés pour épouser le corps mais avec une qualité supérieure, l'objectif étant que ceux éloignés des corps soient proches de maillages cartésiens non uniformes. Cette évolution permet à la fois de pouvoir modéliser des géométries plus complexes mais surtout d'améliorer la résolution. Pour ce faire, un traitement spécial est réalisé à l'interface entre les blocs (Fig. 3).

Comme on travaille avec des blocs structurés, chaque face met en contact uniquement 2 blocs et il est possible d'adopter les discrétisations utilisées précédemment (un nœud à l'intérieur de la face est résolu 2 fois mais avec la même discrétisation). Par contre, aux arêtes et aux nœuds des blocs (Fig. 4), la difficulté vient à la fois du nombre de blocs voisins qui est inconnu du fait de la présence de O-grid (structure multi-blocs conforme permettant d'adapter le maillage à la paroi sans propager les couches limites) et du type de discrétisation (conditions aux limites? Équations de Navier Stokes ?). Afin de généraliser le traitement des arêtes et des nœuds, une simple interpolation à partir des voisins directs est effectuée.

\subsection{Parallélisation du code multi-blocs}

Lors de la parallélisation du code de calcul, nous avons choisi d'utiliser une bibliothèque existante comme PETSc ou libMesh permettant de choisir différentes méthodes de résolution ainsi que le préconditionnement utilisé, tout en

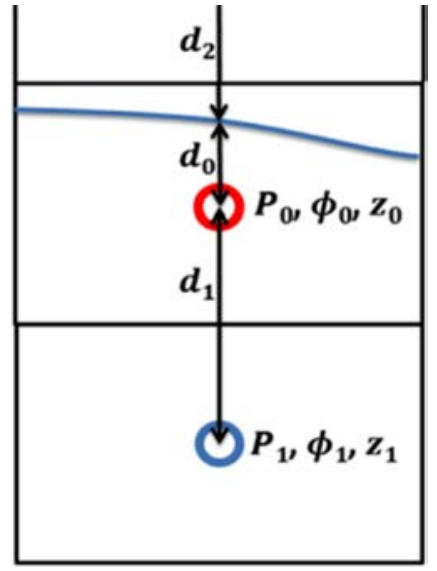

Fig. 2. Traitement de la pression au voisinage de l'interface.
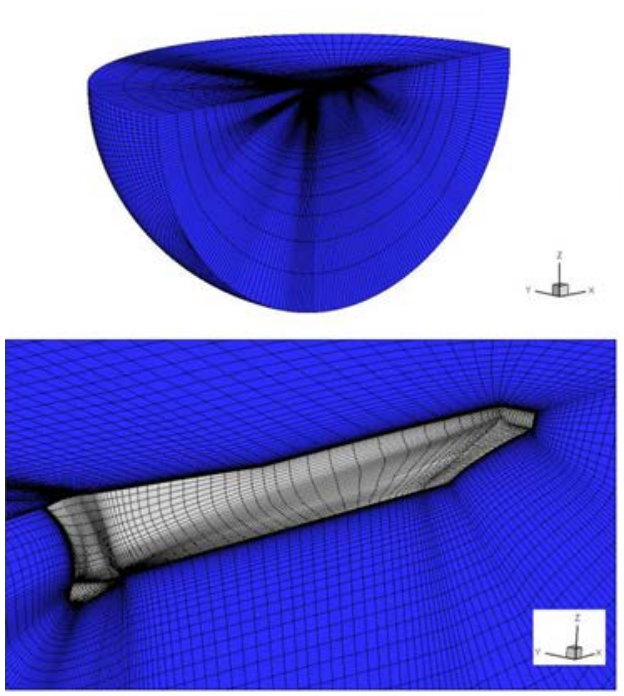

Maillage monobloc

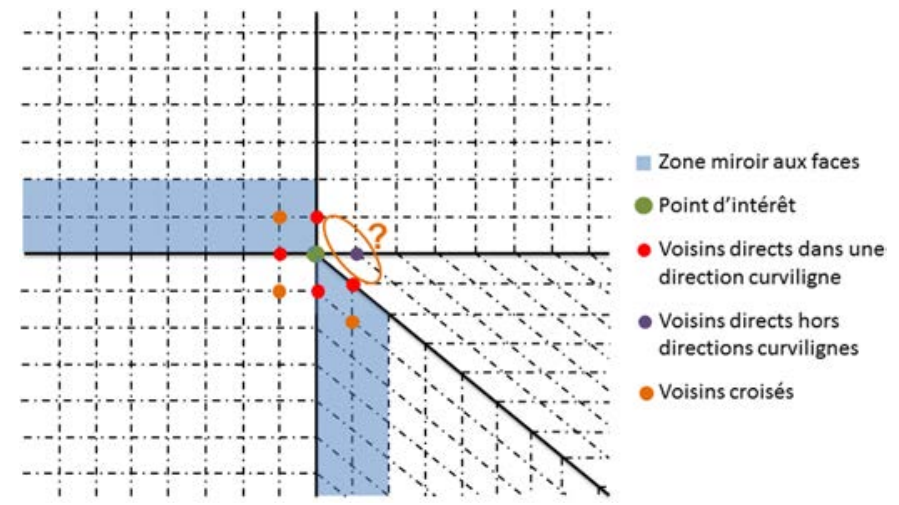

Fig. 4. Voisinage direct aux nœuds à un coin d'un bloc.
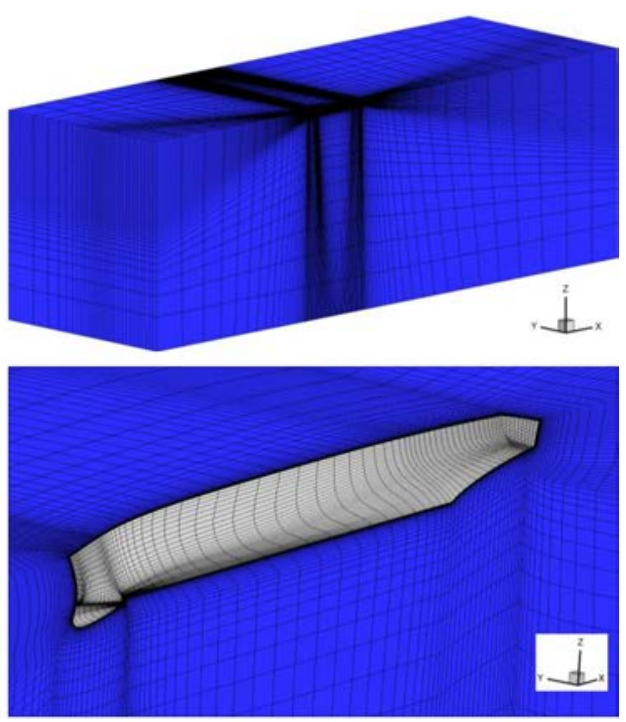

Maillage multi-blocs

Fig. 3. Comparaison des maillages obtenus en monobloc et multi-blocs. 
Tableau 1. Différents types d'équations pour les inconnues aux nœuds.

\begin{tabular}{ll}
\hline $\begin{array}{l}\text { Type de condition } \\
\text { aux limites }\end{array}$ & Équation(s) résolue(s) \\
\hline Nœud intérieur & $\begin{array}{l}\text { Équations de conservation de la } \\
\text { quantité de mouvement }\end{array}$ \\
Surface libre & $\begin{array}{l}\text { Équations obtenues à partir des } \\
\text { conditions de surface libre }\end{array}$ \\
Condition de Dirichlet & $\begin{array}{l}U_{i}=V_{\text {imposée }} \\
\text { Condition de Neumann }\end{array}$ \\
Nœuds et arêtes & Interpolation avec les voisins directs \\
\hline
\end{tabular}

Tableau 2. Propriétés du catamaran Delft 372.

\begin{tabular}{llc}
\hline Propriétés & Symbole & Valeur \\
\hline Longueur entre perpendiculaires & $L p p$ & $3,0 \mathrm{~m}$ \\
Largeur & $B$ & $0,94 \mathrm{~m}$ \\
Largeur d'une demi-carène & $B$ & $0,24 \mathrm{~m}$ \\
Distance entre les centres des flotteurs & $H$ & $0,70 \mathrm{~m}$ \\
Tirant d'eau & $T$ & $0,15 \mathrm{~m}$ \\
Déplacement & $D$ & $87,07 \mathrm{~kg}$ \\
Position verticale du centre de gravité & $K G$ & $0,34 \mathrm{~m}$ \\
Position longitudinale du centre de gravité & $L C G$ & $1,41 \mathrm{~m}$ \\
Rayon de giration en tangage & $K y y / L p p$ & 0,26 \\
Profondeur du bassin & $H$ & $6,50 \mathrm{~m}$ \\
\hline
\end{tabular}

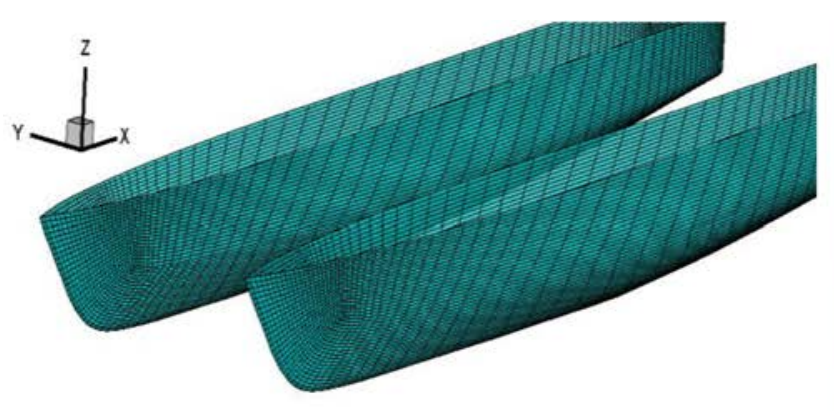

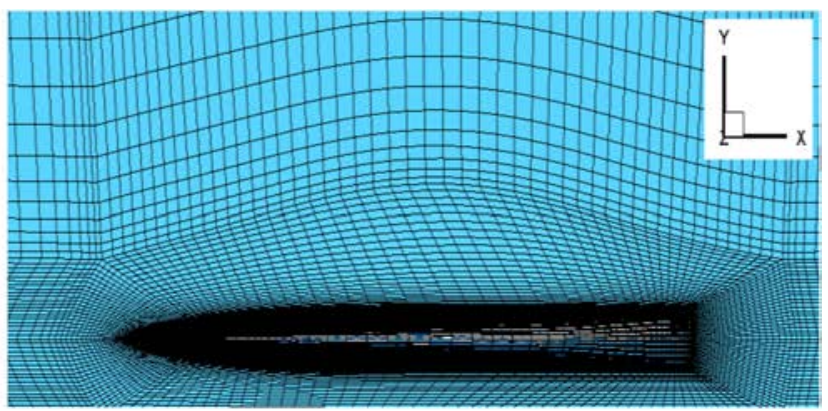

Fig. 5. Vue du maillage sur le corps et au niveau de la surface libre.

intégrant la librairie MPI (Message Passing Interface) pour les communications entre cœurs. Compte tenu des spécificités du maillage (multi-blocs + particularité du traitement aux arêtes et aux faces), le choix a été fait de retenir la librairie PETSc (Balay et al., 1997; Balay et al., 2016). Le maillage multiblocs est vu par Petsc comme un maillage déstructuré et, par conséquent, chaque nœud (même s'il est commun à plusieurs blocs) est traité une seule fois (Tab. 1).

Aux nœuds, les différentes équations résolues peuvent être regroupées en 5 types comme indiqué dans le tableau cidessus. Ce travail de généralisation a permis de simplifier grandement le code puisque auparavant chaque condition était programmée spécifiquement.

Aux centres des cellules, la seule difficulté vient du nombre de cellules voisines intervenant lors de la discrétisation de l'équation de pression à l'aide de la reconstruction de Rhie et Chow. Il faut bien voir qu'avec le positionnement entrelacé partiel adopté, le nombre de coefficients non nuls sur une ligne de la matrice pour la pression est supérieur à 43 (le point d'intérêt, 18 voisins au moins pour la pression et 24 pour la discrétisation de la divergence de la vitesse) contre au moins 10 pour une ligne pour les vitesses et 13 pour les pseudo-vitesses (contribution dans les équations de quantité de mouvement des termes relatifs aux nœuds voisins du nœud considéré). Comme on résout un système couplé vitesses-pression, la matrice comprend environ 1,4 million de lignes pour un maillage de 200000 nœuds. Avec un tel nombre de coefficients non nuls par ligne, seul un préconditionnement simple est possible car sinon la mémoire nécessaire est trop importante. La seule solution serait alors de résoudre de manière découplée à l'aide d'une méthode de type PISO. Toutefois, on préfère conserver l'écriture d'un système couplé qui permet de converger plus rapidement à chaque pas de temps.

\section{Résultats}

Dans la suite, des résultats de simulation avec le catamaran Delft 372 (Tab. 2) sont comparés aux données numériques et expérimentales disponibles dans la littérature. On traite dans un premier temps un cas sur mer calme, puis un cas sur houle régulière pour deux nombre de Froude et enfin un cas sur houle irrégulière. Dans la littérature, on trouve des essais pour différentes distances entres les centres des flotteurs mais on s'intéresse ici uniquement à la configuration $H / L p p=0,23$ car elle correspond à celle utilisée lors des essais sur houle (Fig. 5).

Un maillage multi-blocs d'un demi-domaine a été réalisé avec le logiciel de maillage ICEM, puis symétrisé pour obtenir un maillage complet de 800000 cellules environ. Les calculs sont lancés sur 6 cœurs (pour un maillage multi-blocs constitués de 48 blocs structurés).

\subsection{Catamaran Delft 372 sur mer calme}

Pour les cas sur mer calme, on considère 8 vitesses $\left(F r=\frac{U}{\sqrt{g L p p}}\right)$ entre $F r=0,15$ et $F r=0,7$ en s'intéressant particulièrement à la zone $F r=0,30$ à $F r=0,5$. On compare les résultats obtenus aux résultats fournis dans les articles de la littérature (Castiglione et al., 2011; Visonneau et al., 2012). Les simulations montrent un comportement correct de 


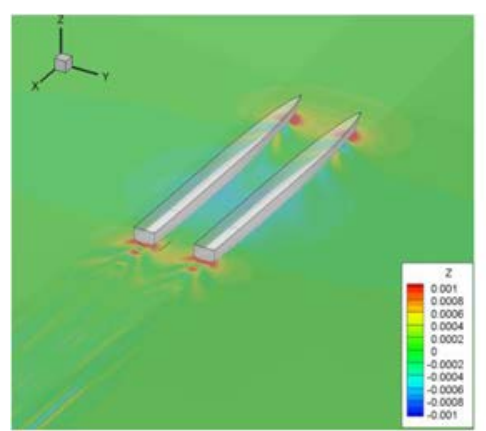

$\mathrm{Fr}=0,15$

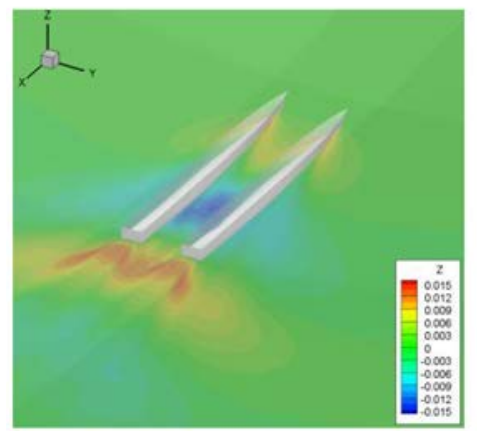

$\mathrm{Fr}=0,40$

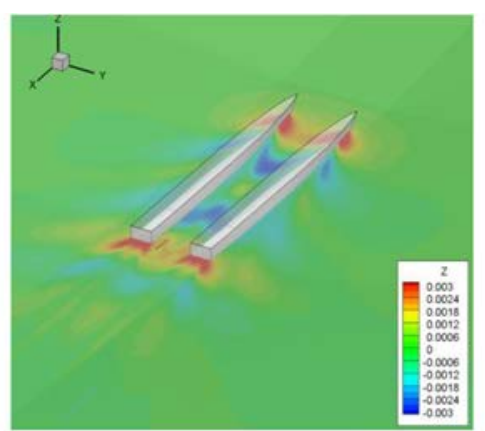

$\mathrm{Fr}=0,25$

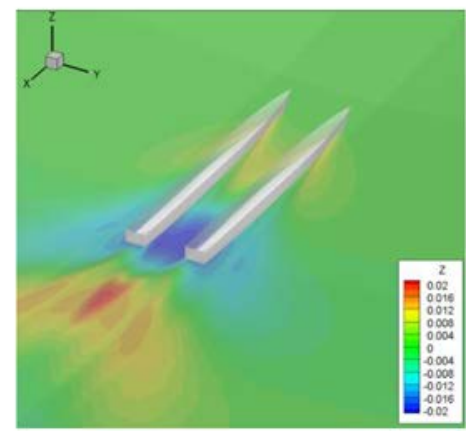

$\mathrm{Fr}=0,5$

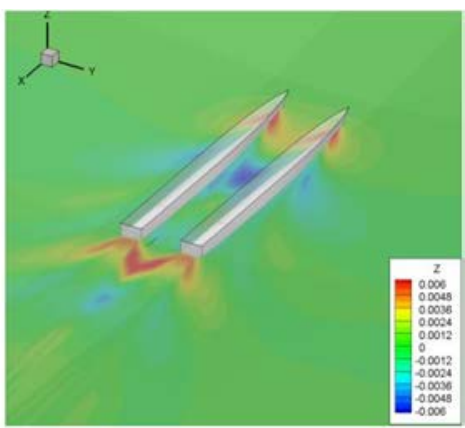

$\mathrm{Fr}=0,30$

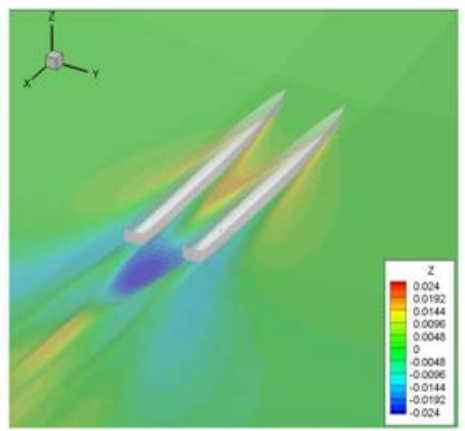

Fr $=0,6$

Fig. 6. Évolution de la surface libre proche du corps en fonction du nombre de Froude Fr.

Tableau 3. Différences entre les bassins.

\begin{tabular}{|c|c|c|c|}
\hline Bassin & $\mathrm{BSHC}$ & TU Delft & Insean \\
\hline $\begin{array}{l}\text { Rapport profondeur du bassin } \\
\text { sur tirant d'eau }\end{array}$ & 8,2 & 17,3 & 43,3 \\
\hline $\operatorname{Re}\left(\times 10^{6}\right)$ & 8,34 & 5,36 & 7,72 \\
\hline $\begin{array}{l}\text { Position verticale du centre } \\
\text { de gravité/Lpp }\end{array}$ & 0,05 & 0,063 & 0,063 \\
\hline
\end{tabular}

l'évolution de la surface libre autour du catamaran avec un déplacement des creux et des crêtes du champ de vagues vers l'arrière du Delft 372 ainsi qu'un accroissement de leurs amplitudes au fur et à mesure de l'augmentation du nombre de Froude (Fig. 6).

La figure 7 présente l'évolution du coefficient de trainée totale, de l'enfoncement dynamique (en mm) et de l'assiette (en degré) pour les différentes vitesses étudiées. Les termes EFD (Experimental Fluid Dynamics) et CFD (Computational Fluid Dynamics) désignent respectivement les essais en bassin et les calculs numériques. Compte tenu des disparités des résultats expérimentaux qui s'expliquent par les différences entre les essais (maquette, bassin... Tab. 3), les résultats sont très satisfaisants surtout si on tient compte de la faible densité du maillage comparée à ceux utilisés par les autres codes de calcul.

\subsection{Catamaran Delft 372 à $F r=0,3$ sur houle régulière}

Pour obtenir la RAO (Response Amplitude Operator) du catamaran Delft 372 aux deux nombres de Froude étudiés, on se limitera aux cinq houles régulières dont les caractéristiques sont données ci-dessous (Tab. 4).

$\mathrm{Si}$ on s'intéresse aux RAOs en pilonnement et tangage données sur la figure 8 , c'est-à-dire aux valeurs des premiers harmoniques adimensionnalisés respectivement par l'amplitude de la houle et la cambrure, on voit apparaître des différences importantes autour de la résonance. Toutefois, en se comparant aux résultats numériques présentés dans (Castiglione et al., 2011), on observe un bon accord entre nos simulations et le code RANS différences finies CFD ShipIowa pour le tangage, les résultats étant quasi-confondus après $\lambda / L p p=1$. Pour le pilonnement, on observe la même tendance pour les deux codes avec une sous-estimation du pic observé autour de $\lambda / L p p=1$ et un comportement correct aux grandes longueurs d'onde. Les courbes étant directement extraites de l'article, les résultats obtenus avec VERES sont présentés. Le code VERES, comme la plupart des codes basés sur la théorie des tranches, prédit globalement bien la réponse du navire mais surestime l'amplitude de la résonance.

\subsection{Catamaran Delft 372 à $F r=0,45$ sur houle régulière}

Là encore, cinq configurations sont testées (Tab. 5) afin de se comparer aux résultats obtenus par l'IIHR avec le code CFDShip IOWA (Fig. 9).

Pour ce second cas, on obtient un très bon accord entre nos simulations et les résultats expérimentaux pour la RAO en pilonnement. En particulier, on arrive à capter très bien le pic en pilonnement qui est observé autour de $\lambda / L p p=1,2$. Pour la RAO en tangage, le maximum trouvé par les simulations est sous-estimé par rapport au maximum des expériences. De plus, le tangage pour les grandes longueurs d'onde est sous-estimé de $8 \%$ environ par l'approche numérique. 

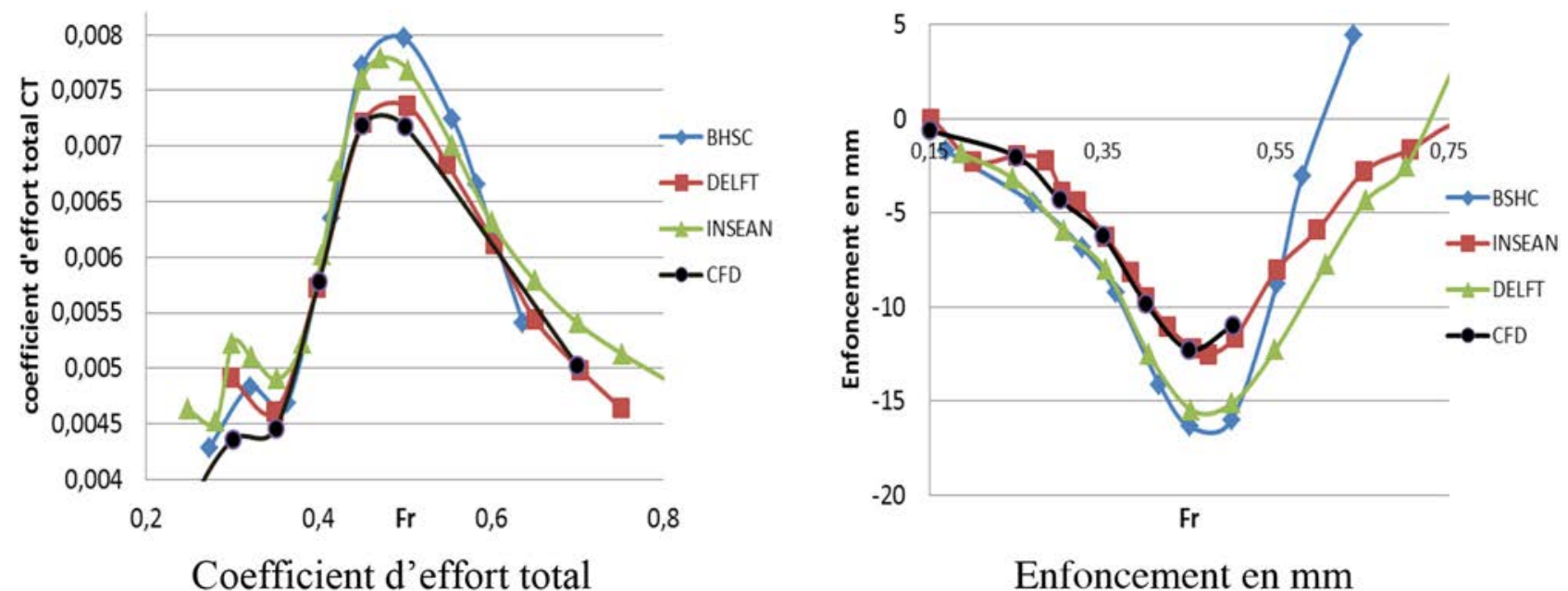

Enfoncement en mm

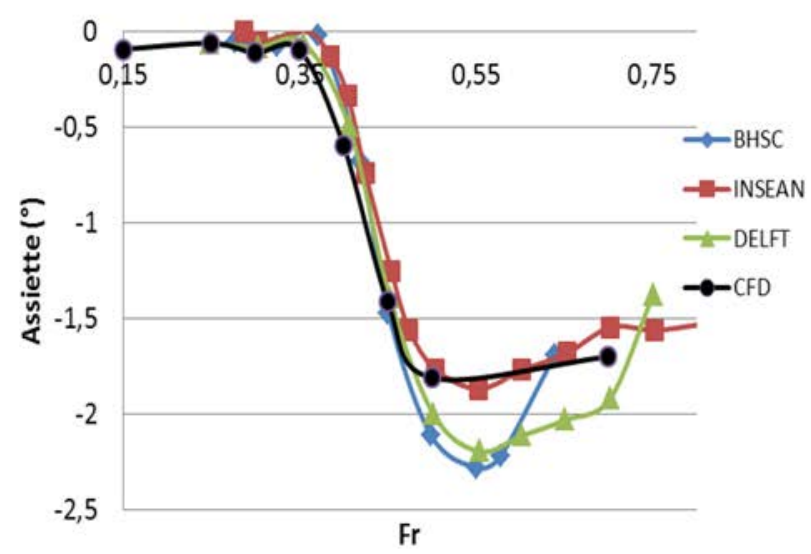

\section{Assiette en degré}

Fig. 7. Comparaison de l'évolution du coefficient d'effort total et de la position dynamique de la carène avec les expériences et des calculs en fonction du nombre de Froude.

Tableau 4. Paramètres des 5 houles réalisées à $F r=0,3$.

\begin{tabular}{|c|c|c|c|c|}
\hline $\begin{array}{l}\text { Cambrure } \\
A k\end{array}$ & $\lambda / L p p$ & $\begin{array}{l}\text { Longueur d'onde } \\
\lambda(\mathrm{m})\end{array}$ & $\begin{array}{l}\text { Nombre d'onde } \\
k\left(\mathrm{~s}^{-1}\right)\end{array}$ & $\begin{array}{l}\text { Elévation crête à creux } \\
h(\mathrm{~m})\end{array}$ \\
\hline 0,0427 & 0,899 & 2,697 & 2,3296 & 0,0366 \\
\hline 0,0447 & 0,965 & 2,895 & 2,1703 & 0,0411 \\
\hline 0,0323 & 1,204 & 3,612 & 1,7395 & 0,0371 \\
\hline 0,0288 & 1,301 & 3,903 & 1,6098 & 0,0357 \\
\hline 0,0219 & 1,803 & 5,409 & 1,1616 & 0,0377 \\
\hline
\end{tabular}

Tableau 5. Paramètres des 5 houles réalisées à $F r=0,45$.

\begin{tabular}{|c|c|c|c|c|}
\hline $\begin{array}{l}\text { Cambrure } \\
A k\end{array}$ & $\lambda / L p p$ & Longueur d'onde $\lambda(\mathrm{m})$ & $\begin{array}{l}\text { Nombre d'onde } \\
k\left(\mathrm{~s}^{-1}\right)\end{array}$ & $\begin{array}{l}\text { Elévation crête à creux } \\
h(\mathrm{~m})\end{array}$ \\
\hline 0,038 & 1,001 & 3,003 & 2,0923 & 0,0363 \\
\hline 0,031 & 1,201 & 3,603 & 1,7438 & 0,0355 \\
\hline 0,027 & 1,396 & 4,188 & 1,5002 & 0,0359 \\
\hline 0,022 & 1,595 & 4,785 & 1,3131 & 0,0335 \\
\hline 0,019 & 1,991 & 5,973 & 1,0519 & 0,0361 \\
\hline
\end{tabular}



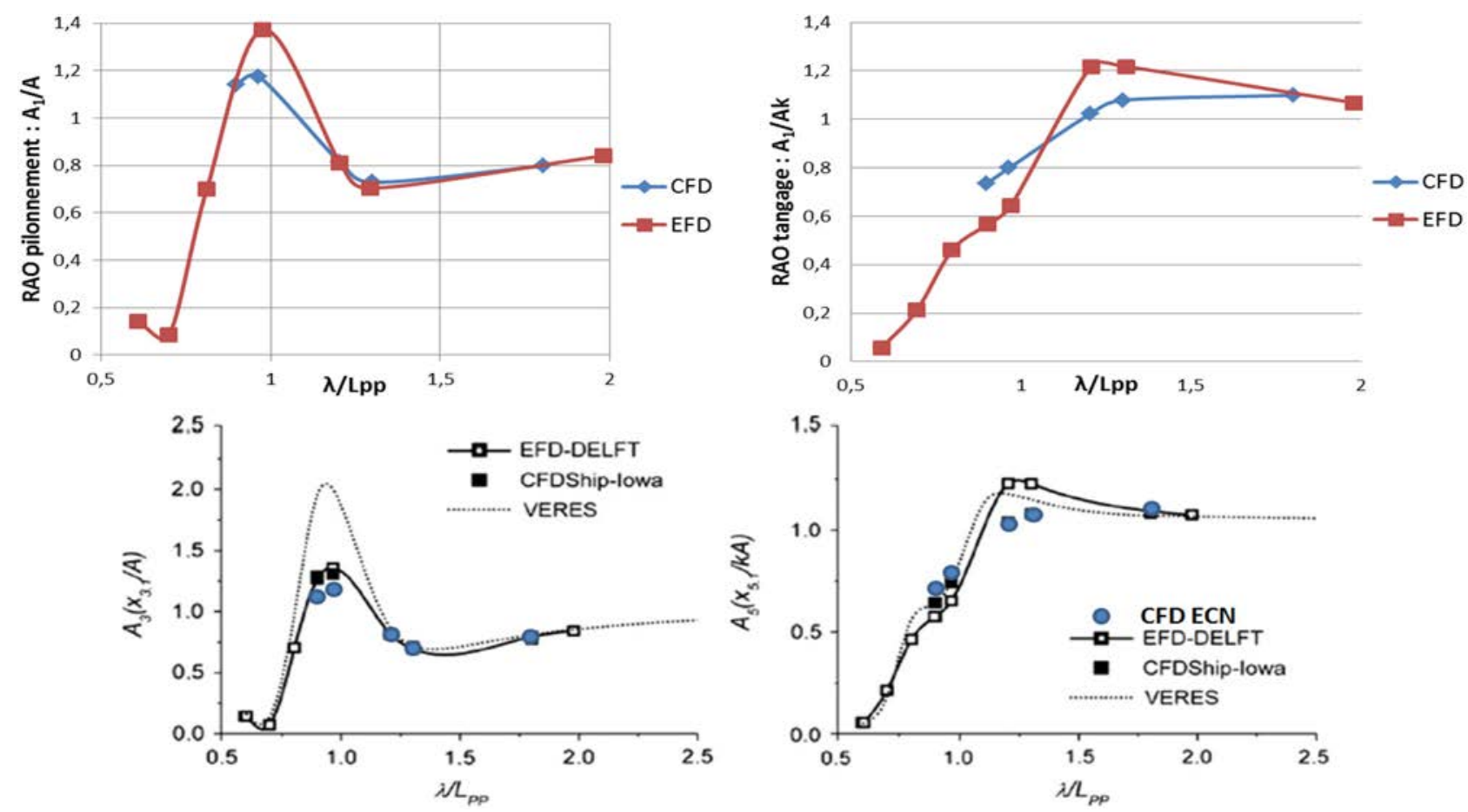

Fig. 8. RAO en pilonnement et tangage pour le catamaran Delft 372 à $\mathrm{Fr}=0,3$.
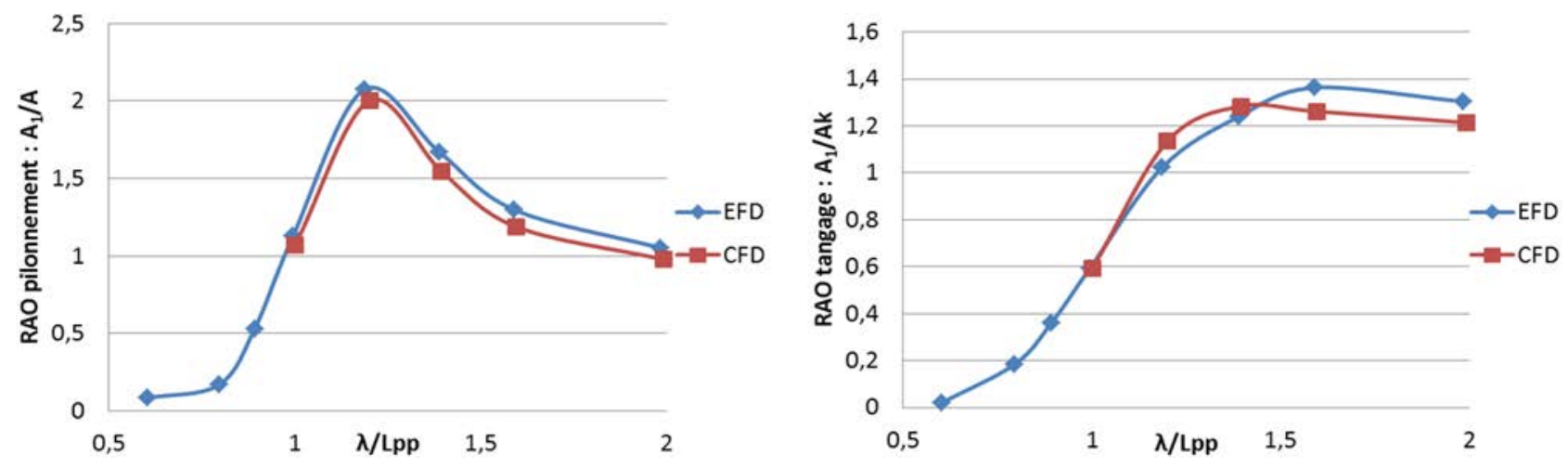

Fig. 9. RAOs en pilonnement et tangage pour le catamaran Delft 372 à $\mathrm{Fr}=0,45$.

\section{Conclusion}

Ce papier présente les développements récents du code multi-blocs basé sur ICARE. L'utilisation des topologies multiblocs a permis d'améliorer la résolution par une meilleure qualité des maillages. La parallélisation, quant à elle, a rendu possible une diminution du temps de restitution grâce à l'utilisation de la librairie PETSc, retenue pour sa flexibilité.

Des cas de validations ont été réalisés sur le catamaran Delft 372. Ce modèle a été utilisé car de nombreuses expériences et simulations sont disponibles aussi bien sur mer calme que sur houle régulière. Les résultats montrent à la fois un bon accord avec les expériences mais aussi avec d'autres codes de calcul.

\section{Références}

Adalsteinsson D, Sethian J. 1999. The fast construction of extension velocities in Level Set methods. J Comput Phys 148: 2-22.

Balay S, Gropp WD, McInnes LC, Smith BF. 1997. Efficient management of parallelism in object oriented numerical software libraries. Modern software tools in scientific computing. Birkhäuser Press, pp. 163-202.

Balay S, Abhyankar S, Adams MF, Brown J, Brune P, Buschelman K, Dalcin L, Eijkhout V, Gropp WD, Kaushik D, Knepley MG, et al. 2016. PETSc users manual. Argonne National Laboratory.

Castiglione T, Stern F, Bova S, Kandasamy M. 2011. Numerical investigation of the seakeeping behavior of a catamaran advancing in regular head waves. Ocean Engineering 38: 1806-1822. 
Drouet A. 2011. Apports de la résolution mutli-blocs pour la simulation de la manœuvrabilité des sous-marins et des bâtiments de surface. Thèse de doctorat, École Centrale de Nantes.

Ferrant P, Gentaz L, Alessandrini B, Le Touzé D. 2003. A potential/ RANSE approach for regular water wave diffraction about 2D structures. Ship Technol Res 50: 165-171.

He W, Castiglione T, Kandasamy M, Stern F. 2015. Numerical analysis of the interference effects on resistance, sinkage and trim of a fast catamaran. J Marine Sci Technol 20(12): 292-308.

Luquet R. 2007. Simulation numérique de l'écoulement visqueux autour d'un navire soumis à une houle quelconque. Thèse de doctorat, École Centrale de Nantes.

Monroy C. 2010. Simulation numérique de l'interaction houlestructure en fluide visqueux par décomposition fonctionnelle. Thèse de doctorat, École Centrale de Nantes.

Reliquet G. 2013. Simulation numérique de l'interaction houle/ carène par couplage d'une méthode spectrale HOS avec un algorithme de capture d'interface. Thèse de doctorat, École Centrale de Nantes.

Reliquet G, Robert M, Drouet A, Guillerm P, Gentaz L, Ferrant P. 2014. Application du couplage SWENSE-Level Set aux simulations de navires sur houles complexes. $14^{e}$ Journées de l'Hydrodynamique. Val de Reuil.

Rhie C, Chow W. 1983. A numerical study of the turbulent flow past an isolated airfoil with trailing edge separation. AIAA J 21: 179-195.

Visonneau M, Deng G, Queutey P, Wackers J, Mallol B. 2012. Anisotropic grid adaptation for RANS simulation of a fast manoeuvring. 4th High Performance Yacht Design Conference. Auckland.

Vukčević V, Jasak H, Malenica Š. 2016. Decomposition model for naval hydrodynamic applications. Part I: computational method. Ocean Engineering 121: 37-46.

Wilcox D. 1988. Reassessment of the scale determining equation for advance turbulence models. AIAA $J$ 26: 1299-1310. 